\title{
A FREQUENCY DOMAIN METHOD FOR CHANNEL ESTIMATION IN MULTIRATE COMMUNICATION SYSTEMS
}

\author{
Hongbo Yan and Sumit Roy \\ Dept. of Electrical Engineering \\ University of Washington, Box 352500 \\ Seattle, WA 98195
}

\begin{abstract}
In this paper, a new frequency domain approach towards blind channel identification for multirate communication systems is described. Users are first separated based on different cyclic frequencies corresponding to their respective symbol rates, thereby resulting in a single-user (blind) identification scenario. The algorithm proposed in [3] is then used to estimate the channels for each rate. Computer simulations demonstrate the effectiveness of our method.
\end{abstract}

\section{INTRODUCTION}

The primary challenge for 3rd generation wireless communications systems is the provisioning of multimedia services (voice, video and data) over the same network infrastructure. Since the rates of such traffic are inherently different, it leads to transceiver design for $m u l$ tirate communications, as distinct from the primarily single-rate scenarios that dominate the literature. For broadband access, Code Division Multiple Access (CDMA) provides a flexible approach to providing multirate services [1] - one of the first techniques for blind channel estimation for multirate CDMA systems was developed in [2]. In this work, we concentrate instead on channel estimation for a generic multirate system model that encompasses both narrowband and wideband signals; such models apply to the case of system overlay where a new wideband service overlaps a legacy narrowband system. Communication signals that are (wide-sense) cyclostationary can be represented by an equivalent single-input, multiple output (SIMO) linear model under output oversampling and/or with multiple receive antennas. It is known that in such cases, it is possible to estimate the channel blindly with second order statistics subject to suitable identifiability conditions [3]-[7]. Our method uses second order cyclic

This work was supported in part by in part by AFOSR Grant F49620-1-0472 statistics to separate multirate users and subsequently applies the approach in [3] for individual channel estimation.

\section{PROBLEM FORMULATION}

A baseband multirate communication system can be modeled as:

$$
y(t)=\sum_{i=1}^{M} \sum_{k=-\infty}^{\infty} s_{i}(k) h_{i}\left(t-k T_{i}\right)+w(t)
$$

where $s_{i}(k)$ 's are mutually independent zero-mean $i . i . d$. sequences with variance $\sigma_{s_{i}}^{2}, M$ is the number of symbol rates and $w(t)$ is additive white noise. Note that we assume only one user at any rate to underscore that rate-based separation is the motivating principle behind our approach. isfies

We assume that the ratio between these rates sat-

$$
\frac{T_{1}}{p_{1}}=\frac{T_{2}}{p_{2}}=\cdots=\frac{T_{M}}{p_{M}}=T
$$

where $p_{1}, p_{2}, \cdots, p_{M}$ are co-prime integers, and $\frac{1}{T}$ is the 'basic' rate. Oversampling the received signal with a factor $\Delta=\frac{T}{L}$ yields

$$
y(n \Delta)=\sum_{i=1}^{M} \sum_{k=-\infty}^{\infty} s_{i}(k) h_{i}\left(n \Delta-k T_{i}\right)+w(n \Delta)
$$

Denote $P=\prod_{i=1}^{M} p_{i}$, the least common multiple of $p_{1}, p_{2}, \cdots, p_{M}$ and $q_{i}=\frac{P}{p_{i}}$, we obtain the discrete time model from (3):

$$
y(n)=\sum_{i=1}^{M} \sum_{k=-\infty}^{\infty} s_{i}(k) h_{i}\left(n-k L p_{i}\right)+w(n),
$$

The problem addressed in this paper is the blind estimation of $h_{i}(n)$ 's. 


\section{BLIND CHANNEL IDENTIFICATION}

The algorithm proposed in this section exploits the finite support property of the second order cyclic statistics (SOCS). Since signals with different cyclic periods have different support, evaluating the SOCS at specifically chosen frequencies leads to separation of individual users, i.e., rejection of multi-user interference, leading to the familiar single user setting.

\subsection{Cyclostationarities of Multirate Signals}

Following the notations and procedures in [8], we first briefly introduce the second order cyclic statistics for the multirate communication signals. The autocorrelation of noiseless received signal $y(n)$ is given by

$$
\begin{aligned}
& r_{y}[n, n+m]=E\left\{y(n) y^{*}(n+m)\right\} \\
& =\sum_{i=1}^{M} \sigma_{s_{i}}^{2} \sum_{k=-\infty}^{\infty} h_{i}\left(n-k L p_{i}\right) h_{i}^{*}\left(n+m-k L p_{i}\right)
\end{aligned}
$$

It is easy to verify that $r_{y}[n, n+m]$ is a periodic function in $n$ with fundamental period $K=L P$, its Fourier expansion is

$$
r_{y}[n, n+m]=\sum_{k=0}^{K-1} R_{y}^{k \alpha}[m] e^{j k n \alpha} \quad \alpha=2 \pi / K
$$

where the cyclic autocorrelation function $R_{y}^{k \alpha}[m]$ is defined as

$$
R_{y}^{k \alpha}[m]=\sum_{n=0}^{K-1} r_{y}[n, n+m] e^{-j k n \alpha}
$$

The spectral correlation density of $y[n]$ is defined as the Fourier transform of $R_{y}^{k \alpha}[m]$

$$
S_{y}^{k \alpha}(f)=\sum_{m} R_{y}^{k \alpha}[m] e^{-j 2 \pi f m}
$$

The support set of these two functions is

$$
U=\{k \alpha \mid k=0, \cdots, K-1\}
$$

Denote by $y_{i}(n)=\sum_{k=-\infty}^{\infty} s_{i}(k) h_{i}\left(n-k L p_{i}\right)$, the component of $y(n)$ due to rate $i$ user. We see that $y_{i}(n)$ is also cyclostationary and the support of $R_{y_{i}}^{k \alpha}[m]$ and $S_{y_{i}}^{k \alpha}(f)$ is

$$
U_{i}=\left\{k q_{i} \alpha=2 \pi k / L p_{i} \mid k=0, \cdots, L p_{i}-1\right\}
$$

where obviously $U_{i} \subset U$.

\subsection{The Algorithm}

Let $\beta=k \alpha$ for brevity; it is easy to verify from (7) and (8) that

$$
R_{y}^{\beta}(m)=\sum_{i=1}^{M} R_{y_{i}}^{\beta}(m), \quad S_{y}^{\beta}(f)=\sum_{i=1}^{M} S_{y_{i}}^{\beta}(f)
$$

Hence for $\beta \in \tilde{U}_{i}=\left\{x \mid x \in U_{i}, x \notin \cup_{j \neq i} U_{j}\right\}$,

$$
S_{y}^{\beta}(f)=S_{y_{i}}^{\beta}(f)
$$

since all other signal components' contribution to $R_{y}^{\beta}(m)$ is identically zero.

Thus we reach the conclusion that when operating on the set $\tilde{U}_{i}$, we are actually dealing with single rate systems from the viewpoint of cyclostationarity. In the following, we therefore consider the signal rate case [3] only.

Evaluate the spectral correlation density of $y(n)$ at $\beta \in \tilde{U}_{i}$

$$
S_{y}^{\beta}(f)=S_{y_{i}}^{\beta}(f)=\sigma_{s_{i}}^{2} H_{i}\left(e^{j(2 \pi f-\beta)}\right) H_{i}^{*}\left(e^{j 2 \pi f}\right)
$$

where $H_{i}\left(e^{j 2 \pi f}\right)$ is the Fourier transform of $h_{i}(n)$. In $Z$-domain, the corresponding formula of (13) is

$$
S_{y}^{\beta}(z)=S_{y_{i}}^{\beta}(z)=\sigma_{s_{i}}^{2} H_{i}\left(z e^{-j \beta}\right) H_{i}^{*}\left(1 / z^{*}\right)
$$

If there are at least two elements in the set $\tilde{U}_{i}$, choose $\underline{\beta}=\left(\beta_{1}, \beta_{2}\right) \in \tilde{U}_{i}$, we can then obtain the following equation:

$$
S_{y}^{\beta_{1}}(z) H_{i}\left(z e^{-j \beta_{2}}\right)=S_{y}^{\beta_{2}}(z) H_{i}\left(z e^{-j \beta_{1}}\right)
$$

According to (8), (15) is equivalent to the following matrix form

$$
\mathbf{R}_{\boldsymbol{y}}(\underline{\beta}) \mathbf{h}_{i}=\mathbf{0}
$$

where $\mathbf{h}_{i}=\left[h_{i}(0) \cdots h_{i}\left(N_{i}\right)\right]^{T}, N_{i}$ is the order of channel $h_{i}(n) ; \mathbf{R}_{y}(\underline{\beta})=\mathbf{R}_{y}^{1}(\underline{\beta})-\mathbf{R}_{y}^{2}(\underline{\beta})$ with

$\mathbf{R}_{y}^{1}(\underline{\beta})=\left[\begin{array}{cccc}R_{y}^{\beta_{1}}\left[-N_{i}\right] e^{j \beta_{2} \cdot 0} & & & \\ \vdots & R_{y}^{\beta_{1}}\left[-N_{i}\right] e^{j \beta_{2} \cdot 1} & & 0 \\ R_{y}^{\beta_{1}}\left[N_{i}\right] e^{j \beta_{2} \cdot 0} & \vdots & & \\ & R_{y}^{\beta_{1}}\left[N_{i}\right] e^{j \beta_{2} \cdot 1} & \ddots & R_{y}^{\beta_{1}}\left[-N_{i}\right] e^{j \beta_{2} \cdot N_{i}} \\ \mathbf{0} & & \ddots & R_{y}^{\beta_{1}}\left[N_{i}\right] e^{j \beta_{2} \cdot N_{i}}\end{array}\right]$
$\mathbf{R}_{y}^{2}(\underline{\underline{\beta}})=\left[\begin{array}{cccc}R_{y}^{\beta_{2}}\left[-N_{i}\right] e^{j \beta_{1} \cdot 0} & & \ddots & 0 \\ \vdots & R_{y}^{\beta_{2}}\left[-N_{i}\right] e^{j \beta_{1} \cdot 1} & & 0 \\ R_{y}^{\beta_{2}}\left[N_{i}\right] e^{j \beta_{1} \cdot 0} & \vdots & & \\ & R_{y}^{\beta_{2}}\left[N_{i}\right] e^{j \beta_{1}} \cdot 1 & \ddots & R_{y}^{\beta_{2}}\left[-N_{i}\right] e^{j \beta_{1} \cdot N_{i}} \\ 0 & & \ddots & \vdots \\ & & & R_{y}^{\beta_{2}}\left[N_{i}\right] e^{j \beta_{1} \cdot N_{i}}\end{array}\right]$ 
In the presence of noise, the least square optimization criterion

$$
\mathbf{h}_{i}=\arg \min _{\left\|\mathbf{h}_{i}\right\|=1}\left\|\mathbf{R}_{y}(\underline{\beta}) \cdot \mathbf{h}_{i}\right\|^{2}
$$

leads to the final solution as the right singular vector associated with the minimum singular value of matrix $\mathbf{R}_{y}(\underline{\beta})$. Similarly, we can estimate other user's channels at different rates.

When there are several choices for $\beta$ in $\tilde{U}_{i}$, We expect better performance with these different $\beta$ s combined. Specifically, for a total of $n$ choices $\left(\bar{\beta}_{l}, l=\right.$ $1 \cdots n$ ) in $\tilde{U}_{i}$, forming the matrix

$$
\mathbf{R}_{y}=\left[\mathbf{R}_{y}^{T}\left(\underline{\beta}_{1}\right) \cdots \mathbf{R}_{y}^{T}\left(\underline{\beta}_{n}\right)\right]^{T}
$$

we can replace (16) with

$$
\mathbf{R}_{y} \mathbf{h}_{i}=\mathbf{0}
$$

\subsection{Identifiability}

It is clear that to determine the channels with our method, two requirements need to be satisfied:

\section{User separatability}

2. Identifiablity for single rate, single user systems

The second condition has been well established [3], therefore we have the following statement.

Theorem $1 h_{i}$ 's can be uniquely determined from (17) (up to some scalar) if and only if

1. for the user at each rate, there exist at least two elements $\beta_{1}$ and $\beta_{2}$ satisfying

$$
\beta_{1}, \beta_{2} \in \tilde{U}_{i}, \quad \tilde{U}_{i}=\left\{x \mid x \in U_{i}, x \notin \cup_{j \neq i} U_{j}\right\}
$$

2. none of the transfer function $H_{i}(Z)$ 's has uniformly $\frac{2 \pi}{L p_{i}}$-spaced zeros

Note that the first condition can be easily met by changing the value of $L$.

\section{SIMULATIONS}

A dual rate system with $p_{1}=2, p_{2}=3$ was considered for performance assessment. Choose $L=2$, thus $K=L P=12$. According to (9) and (10), $U=$ $\left\{0, \frac{\pi}{6}, \frac{2 \pi}{6}, \cdots, \frac{11 \pi}{6}\right\}, U_{1}=\left\{0, \frac{\pi}{2}, \pi, \frac{3 \pi}{2}\right\}$ and $U_{2}=\left\{0, \frac{\pi}{3}, \frac{2 \pi}{3}\right.$, $\left.\cdots, \frac{5 \pi}{3}\right\}$. Since $U_{1} \cap U_{2}=\{0, \pi\}, \tilde{U}_{1}=\left\{\frac{\pi}{2}, \frac{3 \pi}{2}\right\}$ and $\tilde{U}_{2}=\left\{\frac{\pi}{3}, \frac{2 \pi}{3}, \frac{4 \pi}{3}, \frac{5 \pi}{3}\right\}$. Hence with $\underline{\beta}=\left(\frac{\pi}{2}, \frac{3 \pi}{2}\right)$, we can identify $\mathbf{h}_{1}$. Similarly, $\mathbf{h}_{2}$ can be estimated with any one (or combination) of the 6 possible $\beta$ s determined by $\tilde{U}_{2}$, namely $\left(\frac{\pi}{3}, \frac{2 \pi}{3}\right),\left(\frac{\pi}{3}, \frac{4 \pi}{3}\right),\left(\frac{\pi}{3}, \frac{5 \pi}{3}\right),\left(\frac{2 \pi}{3}, \frac{4 \pi}{3}\right),\left(\frac{2 \pi}{3}, \frac{5 \pi}{3}\right)$ and $\left(\frac{4 \pi}{3}, \frac{5 \pi}{3}\right)$.

The channels were generated from the two-ray multipath propagation model

$$
h_{i}(t)=\lambda_{1} p_{i}\left(t-\gamma_{1} T_{i}\right)+\lambda_{2} p_{i}\left(t-\gamma_{2} T_{i}\right) \quad i=1,2
$$

where $\lambda_{1}, \lambda_{2}$ are zero-mean complex Gaussian random variables with unit variance in each component (real and imaginary). The path delays parameters $\gamma_{1}, \gamma_{2}$ are random variables uniformly distributed on $[-11] . p_{i}(t)$ is the raised-cosine pulse shaping function with roll-off factor 0.5 and time limited to $4 T_{i}$. The same set of $\lambda_{l}, \gamma_{l}$ $(l=1,2)$ parameters were used for both channels.

100 Monte Carlo runs were conducted to compute normalized root mean square error (NRMSE), the performance measure which is defined as:

$$
N R M S E=\frac{1}{\|\mathbf{h}\|} \sqrt{\frac{1}{J} \sum_{j=1}^{J}\left\|\hat{\mathbf{h}}_{j}-\mathbf{h}\right\|^{2}}
$$

where $\hat{\mathbf{h}}_{j}$ is the $j$ th estimate of vector $\mathbf{h}$. Cyclic autocorrelation functions are obtained from the observation through

$$
R_{y}^{\beta}[m]=\frac{1}{N-m} \sum_{k=1}^{N-m} y[k] y^{*}[k+m] e^{-j k \beta}
$$

The number of symbols used for rate 1 and rate 2 are 4500 and 3000 respectively. The effect of white Gaussian noise and multirate interference on the algorithm behaviors was investigated separately. Fig. 1 and 2 are the respective plots of NRMSE versus SIR and SNR for rate 1 user. It is easy to see that the algorithm combats both noise and multirate interference successfully. For rate 2 user, performance with every $\beta$ pair and their full combination was tested separately. The results are shown in Figs. 3 and 4 , from which we can conclude that the relative performance of the 6 individual $\beta$ pairs varies as a function of SIR/SNR. In the absence of an a-priori choice of $\beta$, combination of more (or all) pairs may be utilized. Our extensive simulations showed that generally the more $\beta$ pairs used, the better the performance at the cost of additional computational complexity arising from SVD on larger matrix.

\section{REFERENCES}

[1] T. Ottosson and A. Svensson, " Multi-rate schemes in DS/CDMA systems," in Proc. IEEE Vehic. Tech. Conf., Chicago, pp.1006-1010, July 1995. 


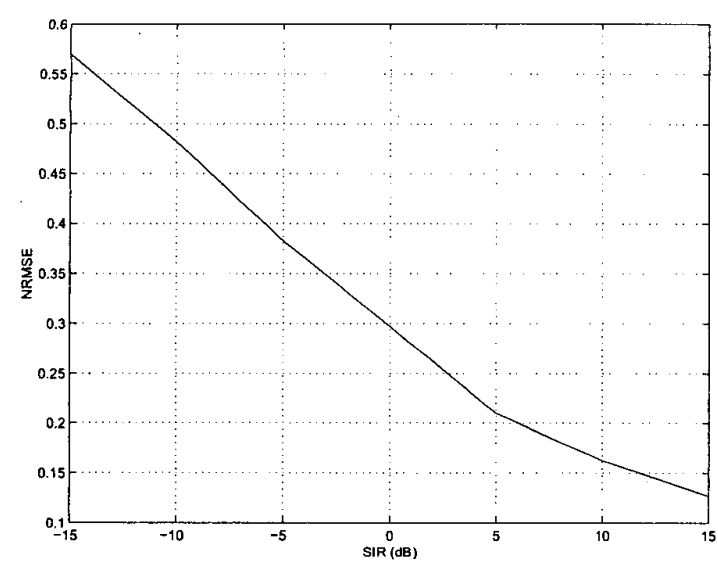

Figure 1: NRMSE versus SIR: rate 1 user

[2] H. Yan and S. Roy, "Blind channel identification for multirate CDMA systems", in Proc. ICASSP vol.5, pp.2509-2512, June 2000.

[3] L. Tong and G. Xu and B. Hassibi and T. Kailath, "Blind channel identification based on secondorder statistics: A frequency domain approach", in IEEE Trans. Inform. Theory, vol.41, no.1 pp.329334, Jan. 1995.

[4] L. Tong and G. Xu and T. Kailath "Blind identification and equalization based on second-order statistics: A time domain approach", in IEEE Trans. Inform. Theory, vol.40, no.3, pp.340-349, Mar. 1994.

[5] L. A. Baccala and S. Roy, "A new blind timedomain channel identification method based on cyclostationarity", in IEEE Signal Processing Letter, vol.1, no.6, pp.89-91, June 1994.

[6] E. Moulines and P. Duhamel and J.-F. Cardoso and S. Mayrargue, "Subspace methods for the blind identification of multichannel FIR filters", in IEEE Trans. Signal Processing, vol.43, no.2, pp.516-525, Feb. 1995.

[7] G. $\mathrm{Xu}$ and H. Liu and L. Tong and T. Kailath, "A Least-Squares Approach to Blind Channel Identification", in IEEE Trans. Signal Processing, vol.43, no.12, pp.2982-2992, Dec. 1995.

[8] W. A. Gardner, "Exploitation of spectral redundancy in cyclostationary signals", in IEEE Signal Processing Mag., vol.8, no.2, pp.14-36, Apr. 1991.

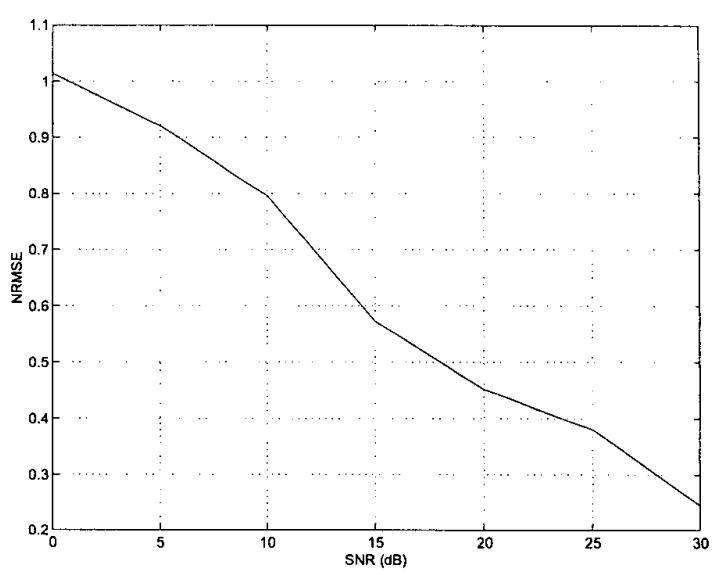

Figure 2: NRMSE versus SNR: rate 1 user, $\mathrm{SIR}=5 \mathrm{~dB}$

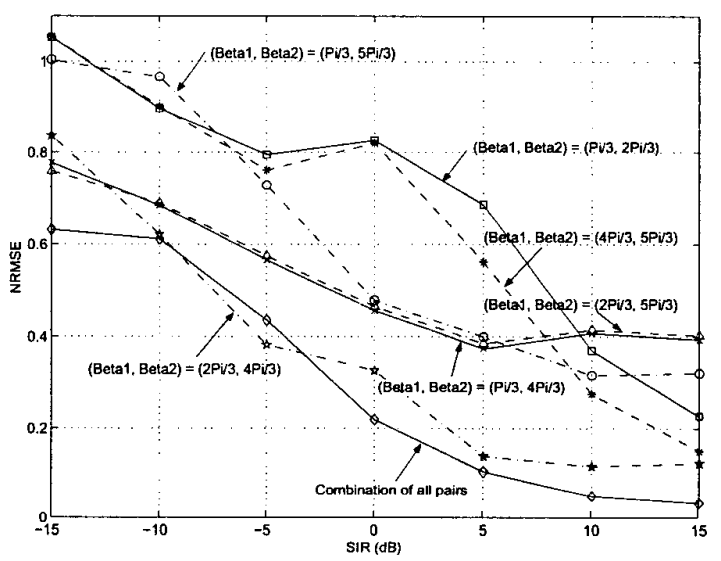

Figure 3: NRMSE versus SIR: rate 2 user

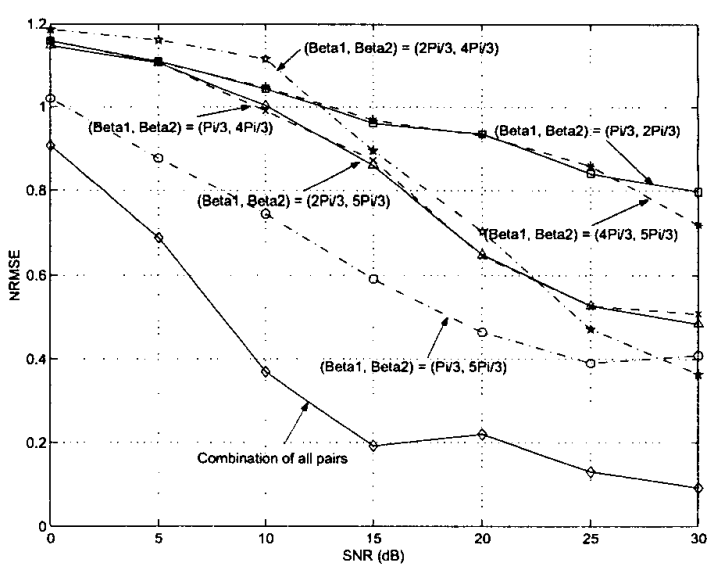

Figure 4: NRMSE versus SNR: rate 2 user, $\mathrm{SIR}=5 \mathrm{~dB}$ 